\title{
OPEN Publisher Correction: Optical clumped isotope thermometry of carbon dioxide
}

\section{Ivan Prokhorov $(\mathbb{D}$, Tobias Kluge \& Christof Janssen (i)}

Correction to: Scientific Reports https://doi.org/10.1038/s41598-019-40750-z, published online 18 March 2019

The original version of this Article contained errors.

References 6-9 were incorrectly listed as references 11-14. References 10-12 were listed as references 7-9. References 13 and 14 were listed as references 15 and 10 respectively. References 15-16 were listed as references 16-17. References 17-23 were listed as references 19-25. References $24-27$ were listed as references $28,26,30$ and 29 respectively. References $28-33$ were listed as references $31-36$.

Reference 34 was listed as reference 27. References 35-36 were listed as references 37 and 39 respectively. References 37-40 were listed as references 40-43. Reference 41 was listed as reference 18 . References $42-52$ were listed as references 44-54. Reference 53 was listed as reference 6. Reference 54 was listed as reference 38.

Furthermore, in the Introduction,

"Typical operation conditions are around $\Delta M / M \sim 40000$ or lower, which is insufficient to separate ${ }^{13} \mathrm{C}^{16} \mathrm{O}_{2}$ from ${ }^{12} \mathrm{C}^{16} \mathrm{O}^{17} \mathrm{O}$ at $\mathrm{m} / \mathrm{z}=45$ or ${ }^{13} \mathrm{C}^{16} \mathrm{O}^{18} \mathrm{O}$ from ${ }^{12} \mathrm{C}^{17} \mathrm{O}^{18} \mathrm{O}$ at $\mathrm{m} / \mathrm{z}=47$, for example."

now reads:

"Typical operation conditions are around $M / \Delta M \sim 40000$ or lower, which is insufficient to separate ${ }^{13} \mathrm{C}^{16} \mathrm{O}_{2}$ from ${ }^{12} \mathrm{C}^{16} \mathrm{O}^{17} \mathrm{O}$ at $\mathrm{m} / \mathrm{z}=45$ or ${ }^{13} \mathrm{C}^{16} \mathrm{O}^{18} \mathrm{O}$ from ${ }^{12} \mathrm{C}^{17} \mathrm{O}^{18} \mathrm{O}$ at $\mathrm{m} / \mathrm{z}=47$, for example."

In the lower inset of Figure 1 the two labels $\mathrm{P}(9)$ and $\mathrm{P}(17)$ were inadvertently switched.

In the Operating Principles and Instrumental Approach section,

"The uncertainty of this calibration is very small: different calculations at $1000 \mathrm{~K}$ are given in the literature $22,33,36$ and our calculation based on partition functions evaluated as direct sums of energy levels provided by ab initio calculations that were refined by spectroscopic measurements ${ }^{34}$, indicate that the error at that temperature is about 5 ppm."

now reads:

"The uncertainty of this calibration is very small: different calculations at $1000 \mathrm{~K}$ are given in the literature ${ }^{22,36}$ and our calculation based on partition functions evaluated as direct sums of energy levels provided by ab initio calculations that were refined by spectroscopic measurements ${ }^{34}$, indicate that the error at that temperature is about 5 ppm."

In Table 2 the column heading,

“CL2012 36 "

now reads:

“CL2012 ${ }^{54 "}$ 
Additionally in Table 2 the column heading,

“CBRZ2014"

now reads:

"CBRZ2014"

Finally, the Table 2 legend,

"Different theories are employed: BMU approach using harmonic frequencies for application of the Teller-Redlich ${ }^{28}$ rule and anharmonic correction to the ZPEs - WSE2004 ${ }^{22}$. The same approach using harmonic frequencies from another level of theory - CL2012 ${ }^{36}$. Path-Integral Monte Carlo (PIMC) evaluation of partition sums - WM2014 ${ }^{33}$. Approximate direct sum partition functions from a refined potential surface - CBRZ2014 ${ }^{54}$. Direct sum calculation of partition functions (this work) using state energies from new spectroscopic data generated from experimentally refined ab initio calculations ${ }^{34}$. aValues at temperatures other than 200,300 and $1000 \mathrm{~K}$ were recalculated from molecular constants in Table 3 of Wang et al. ${ }^{22}$."

now reads:

"Different theories are employed: BMU approach using harmonic frequencies for application of the Teller-Redlich ${ }^{28}$ rule and anharmonic correction to the ZPEs - WSE2004 ${ }^{22}$. The same approach using harmonic frequencies from another level of theory - CL2012 ${ }^{54}$. Path-Integral Monte Carlo (PIMC) evaluation of partition sums - WM2014 $4^{33}$. Approximate direct sum partition functions from a refined potential surface - CBRZ2014 ${ }^{36}$. Direct sum calculation of partition functions (this work) using state energies from new spectroscopic data generated from experimentally refined ab initio calculations ${ }^{34}$. aValues at temperatures other than 200,300 and $1000 \mathrm{~K}$ were recalculated from molecular constants in Table 3 of Wang et al. ${ }^{22}$."

These errors have now been corrected in the PDF and HTML versions of the Article.

Open Access This article is licensed under a Creative Commons Attribution 4.0 International License, which permits use, sharing, adaptation, distribution and reproduction in any medium or format, as long as you give appropriate credit to the original author(s) and the source, provide a link to the Creative Commons license, and indicate if changes were made. The images or other third party material in this article are included in the article's Creative Commons license, unless indicated otherwise in a credit line to the material. If material is not included in the article's Creative Commons license and your intended use is not permitted by statutory regulation or exceeds the permitted use, you will need to obtain permission directly from the copyright holder. To view a copy of this license, visit http://creativecommons.org/licenses/by/4.0/.

(C) The Author(s) 2020 